\title{
Directional Characteristics of The Nonlinear Energy Transfer of Gravity Wave Spectrum
}

\author{
Suciaty FITRI ${ }^{1}$, Noriaki HASHIMOTO${ }^{2}$, Masaki YOKOTA³ ${ }^{3}$ Masaru YAMASHIRO ${ }^{4}$ \\ ${ }^{1} \mathrm{PhD}$ Student, Dept. of Maritime Engineering, Kyushu University \\ (744, Motooka, Nishi-ku, Fukuoka-city, 819-0395, Japan) \\ Email:fitri@civil.kyushu-u.ac.jp \\ 2 Fellow of JSCE, Professor, ditto, Email: hashimoto-n@ civil.kyushu-u.ac.jp \\ 3 Member of JSCE, Assistant Professor, ditto, Email: yokota@ civil.kyushu-u.ac.jp \\ ${ }^{4}$ Member of JSCE, Associate Professor, ditto, Email: yamashiro@ civil.kyushu-u.ac.jp
}

\begin{abstract}
The nonlinear energy transfer plays a critical role in determining the energy distribution of directional spectrum. Numerous studies of the important role of the nonlinear energy transfer in the evolutions of wave spectrum have been clearly demonstrated in the frequency domain, however little is known about the characteristics of the nonlinear energy transfer in the directional domain. In this paper, we investigate the characteristics of spatial and temporal changes of the directional spectra in consideration of nonlinear energy transfer as a possible mechanism of the change of directional distribution in deep water depth. Numerical computations are performed by three different types of the third-generation wave model, WAM, where each of them is implemented with DIA (Hasselmann et. al., 1985), RIAM (Komatsu et. al., 1993) and SRIAM (Komatsu et. al., 1996), respectively, for the computation of the nonlinear energy transfer. The JONSWAP type spectrum and Mitsuyasu's directional function are applied to define the energy distribution of directional spectrum. The characteristics of bimodal directional wave spectra are also discussed.
\end{abstract}

Key Words : directional wave spectrum, nonlinear energy transfer, wave modeling

\section{INTRODUCTION}

The nonlinear energy transfer has been shown to play a key role in the time (fetch) evolution and the establishment of a well-developed wind sea spectrum by controlling the spectral shape, including the development and position of the peak (e.g. Hasselmann et al. 1973). Numerous studies have investigated the importance of the nonlinear energy transfer in the frequency domain. Nevertheless, the characteristics of the nonlinear energy transfer in the directional domain have not been necessarily discussed sufficiently so far. We, therefore, investigate the spatial and temporal characteristics of two-dimensional (directional) spectra by taking into account only the nonlinear energy transfer in deep water depth under various conditions. Studies of two-dimensional spectra of ocean waves are very important, not only for practical purposes such as wave forecasting, but also for clarifying the fundamental process of wave generation and development, etc.

In deep water without currents, the balance equations for the two-dimensional ocean wave spectrum can be written as

$$
\begin{gathered}
\frac{\partial F}{\partial t}+c_{g} \cdot \nabla F=S_{\text {wind }}+S_{n l}+S_{d s} \\
c_{g}=\frac{\partial \omega}{\partial k}, \omega^{2}=g k
\end{gathered}
$$

where $F$ is the two-dimensional wave spectrum; $k$ is the wave number; and $S_{\text {wind }}, S_{n l}$, and $S_{d s}$ represent the various physical process source terms, the wind input, the nonlinear energy transfer, and the dissipation, respectively. Among the three source terms, the nonlinear energy transfer is known as one of the most important factors that control the evolution of wave spectra. 
The nonlinear energy transfer can occur when there is a resonance among four-wave components, that can be expressed with

$$
\begin{aligned}
& \boldsymbol{k}_{1}+\boldsymbol{k}_{2}=\boldsymbol{k}_{3}+\boldsymbol{k}_{4} \\
& \omega_{1}+\omega_{2}=\omega_{3}+\omega_{4}
\end{aligned}
$$

where $\omega_{i}$ is the angular frequency and $\boldsymbol{k}_{i}$ is the wave number vector $(i=1, \ldots, 4)$. The angular frequency $\omega_{i}$ and the wave number $k_{i}$ are related through the dispersion relationship $\left(\omega^{2}=g k_{i} \tanh k_{i} h\right)$.

The nonlinear energy transfer can be written in the following form (Boltzmann integral) (Hasselmann ,1962)

$$
\begin{aligned}
\frac{\partial n\left(\mathbf{k}_{4}\right)}{\partial t} & =\int \cdots \int d \mathbf{k}_{1} d \mathbf{k}_{2} d \mathbf{k}_{3} G\left(\mathbf{k}_{1}, \mathbf{k}_{2}, \mathbf{k}_{3}, \mathbf{k}_{4}\right) \\
& \times \delta\left(\mathbf{k}_{1}+\mathbf{k}_{2}-\mathbf{k}_{3}-\mathbf{k}_{4}\right) \delta\left(\omega_{1}+\omega_{2}-\omega_{3}-\omega_{4}\right) \\
& \times\left\{n_{1} n_{2}\left(n_{3}+n_{4}\right)-n_{3} n_{4}\left(n_{1}+n_{2}\right)\right\}
\end{aligned}
$$

where $n_{i}=n\left(\mathbf{k}_{i}\right)=\Phi\left(\mathbf{k}_{i}\right) / \omega_{i}$ is the wave action density, $\Phi\left(\mathbf{k}_{i}\right)$ is the wave number spectrum, and $G\left(\mathbf{k}_{1}, \mathbf{k}_{2}, \mathbf{k}_{3}, \mathbf{k}_{4}\right)$ is the coupling coefficient.

The third-generation wave models, such as WAM, SWAN, and WW3, adopted DIA by Hasselman et al. (1985) for the nonlinear energy transfer calculation. The DIA method has been known to have low computational cost. Since DIA includes only a low number of configurations of four wave components, this method does not have sufficient accuracy for even standardized wave spectra such as the JONSWAP spectrum.

In the present study, we used the thirdgeneration wave models, original WAM, and modified WAM implemented by RIAM (Komatsu et. al., 1993) and SRIAM (Komatsu et. al., 1996), respectively, for calculating the nonlinear energy transfer. We compared the numerical results by these methods for various directional spectra including multi-modal spectra to know the directional characteristics of the nonlinear energy transfer in ocean wave spectra.

\section{NUMERICAL SIMULATIONS}

Computations of nonlinear energy transfer need an infinite number of possible four-wave configurations to satisfy resonance conditions. It is very important to know how many resonance configurations are required to estimate the nonlinear transfer function for a given wave spectrum. DIA assumes that the interactions between neighboring wave numbers reproduce the principal features of nonlinear transfer (Hasselmann et al. 1985). Komatsu and Masuda (1996) successfully reduced the number of resonant four-wave combinations based on RIAM method with good accuracy. While SRIAM uses 20 resonance configurations with RIAM-based optimization; its computational cost is 100 times less than that of RIAM (20 times more than DIA).

\section{(1) DIA}

The Discrete Interaction Approximation (DIA) was developed by Hasselmann et al. (1985). The full solution to the Boltzmann integral (Eq. 4) in DIA method is using a small number of quadruplets, which all have the same configuration. The resonant conditions are expressed by the following equations:

$$
\begin{aligned}
& \begin{array}{l}
\omega_{1}=\omega_{2}=\omega \\
\omega_{3}=\omega(1+\lambda)=\omega_{+} \quad \theta_{3}-\theta= \pm \cos ^{-1}\left\{\frac{1+2 \lambda+2 \lambda^{3}}{(1+\lambda)^{2}}\right\}
\end{array} \\
& \begin{array}{l}
\omega_{4}=\omega(1-\lambda)=\omega_{-} \\
\theta_{1}=\theta_{2}=\theta
\end{array} \theta_{4}-\theta=\mp \cos ^{-1}\left\{\frac{1-2 \lambda-2 \lambda^{3}}{(1+\lambda)^{2}}\right\}
\end{aligned}
$$

where $\lambda$ is a constant for determining the combination of the component waves. Hasselmann et al (1985) set $\lambda=0.25$ based on numerical experiments. The rates of change of the energy densities $\left(\delta_{n l}, \delta_{n l}{ }^{+}, \delta_{n l}{ }^{-}\right)$with focused only on the configuration and simplified the Boltzmann integral are given by:

$$
\begin{aligned}
& \left\{\begin{array}{l}
\delta S_{n l} \\
\delta S_{n l}^{+} \\
\delta S_{n l}^{-}
\end{array}\right\}=\left\{\begin{array}{l}
-2(\Delta \omega \Delta \theta) /(\Delta \omega \Delta \theta) \\
(1+\lambda)(\Delta \omega \Delta \theta) /\left(\Delta \omega_{+} \Delta \theta\right) \\
(1-\lambda)(\Delta \omega \Delta \theta) /\left(\Delta \omega_{-} \Delta \theta\right)
\end{array}\right\} \\
& \times C \omega^{11} g^{-4}\left[F^{2}\left\{\frac{F_{+}}{(1+\lambda)^{4}}+\frac{F_{-}}{(1-\lambda)^{4}}\right\}-2 \frac{F F_{+} F_{-}}{\left(1-\lambda^{2}\right)^{4}}\right]
\end{aligned}
$$

where $F \equiv F(\omega, \theta), F_{+} \equiv F\left(\omega_{+}, \theta\right), F_{-} \equiv F\left(\omega_{-}, \theta\right)$ are the energy densities at the values of the interacting wave numbers, $g$ is the gravitational acceleration, and $C$ is a constant equal to $3 \times 10^{7}$. 


\section{(2) RIAM}

The RIAM method improves the efficiency of Masuda's (1980) method by utilizing the symmetry characteristic of the Boltzmann integral. Masuda (1980) reduced the six-fold Boltzmann integral to the three-fold integral which be able to reduce the computational time to a large extent as expressed by the following equation:

$$
\begin{aligned}
& \frac{\partial \phi_{4}}{\partial t}=\left(2 \omega_{4}^{23}\right) \int_{0}^{\pi} d \tilde{\theta}_{3} \int_{0}^{\infty} d \tilde{\Omega} \int_{0}^{\pi} d \tilde{\theta}_{1} \\
& \sum_{ \pm} \sum_{ \pm} \sum_{ \pm}\left(8 \tilde{\omega}_{1}^{3} \tilde{\omega}_{3}^{4} \tilde{G} S^{-1}\right)\left\{n_{1} n_{2}\left(n_{3}+n_{4}\right)-n_{3} n_{4}\left(n_{1}+n_{2}\right)\right\}
\end{aligned}
$$

with assuming $\left|\mathbf{k}_{1}\right| \leq\left|\mathbf{k}_{2}\right|$ (or $\omega_{1} \leq \omega_{2}$ ).

The variables are non-dimensionalised by

$$
\begin{array}{ccc}
\tilde{\theta}_{1}=\theta_{1}-\theta_{a}, & \tilde{\theta}_{2}=\theta_{2}-\theta_{a}, & \tilde{\theta}_{3}=\theta_{3}-\theta_{4}, \\
\tilde{\omega}_{1}=\omega_{1} / \omega_{4}, & \tilde{\omega}_{2}=\omega_{2} / \omega_{4}, & \tilde{\omega}_{3}=\omega_{3} / \omega_{4}
\end{array}
$$

and $\tilde{\Omega}=\ln \tilde{\omega}_{3}$.

The denominator $S$ arising from $\delta\left(\omega_{1}+\omega_{2}-\omega_{3}-\omega_{4}\right)$ is given by

$$
S=\left|1+\left(\omega_{1} / \omega_{2}\right)\left\{\left(\omega_{1} / \omega_{2}\right)^{2}-\left(\left|\mathbf{k}_{a}\right| / \omega_{2}^{2}\right) \cos \left(\theta_{1}-\theta_{a}\right)\right\}\right|
$$

Masuda solved the instability problem by analytically deriving an approximate solution of Eq. (9) around the singular points. Komatsu and Masuda (1996) developed RIAM for calculating the nonlinear energy transfer based on Masuda's method by using the symmetry of the integrand as in Hasselmann and Hasselmann (1981) or Resio and Perrie (1991), and by truncating less significant configurations of resonance.

\section{(3) SRIAM}

Komatsu (1996) developed a new scheme with RIAM-based optimization, called the Simplified RIAM (SRIAM) method. The SRIAM method can be expressed by the following equation.

$$
\begin{aligned}
T\left(\omega_{4}, \theta_{4}\right)= & \frac{\partial \phi_{4}}{\partial t}=\left(2 \omega_{4}^{23}\right) \sum_{i=1}^{20} C_{i} \tilde{K}_{i}\left(\tilde{\theta}_{1}, \tilde{\Omega}, \tilde{\theta}_{3}\right) \\
& \times\left\{n_{1} n_{2}\left(n_{3}+n_{4}\right)-n_{3} n_{4}\left(n_{1}+n_{2}\right)\right\}_{i}
\end{aligned}
$$

where $\tilde{K}_{i}\left(\tilde{\theta}_{1}, \tilde{\Omega}, \tilde{\theta}_{3}\right)=8 \tilde{\omega}_{1}^{3} \omega_{3}^{4} \tilde{G} S^{-1} \Delta \tilde{\theta}_{3} \Delta \tilde{\Omega} \Delta \tilde{\theta}_{1}$, and $C_{i}$, $(i=1, \cdots 20)$ are the coefficients.
Journal of Japan Society of Civil Engineers, Ser. B3 (Ocean Engineering), Vol. 71, No. 2, I_347-I_352, 2015.

Komatsu (1996) identified 20 optimized quadruplets that represent the infinite number of possible resonant four-wave configurations, where 7 configurations are chosen for singular points and the other 13 configurations are chosen for regular points from $\left(\tilde{\theta}_{1}, \tilde{\Omega}, \tilde{\theta}_{3}\right)$ space.

\section{RESULTS OF NUMERICAL SIMULATIONS}

Numerical simulations are performed under duration-limited conditions for 12 hours using WAM with DIA, RIAM, and SRIAM methods by taking account of only the nonlinear energy transfer $S_{n l}$, without the wind input $\left(S_{i n}=0\right)$ and the dissipation $\left(S_{d i s}=0\right)$.

\section{(1) Initial Conditions in Numerical Simulations}

In this study, we assumed the directional spectrum as $S(f, \theta)=S(f) G(\theta \mid f)$, where the frequency spectrum $S(f)$ and the directional function $G(\theta \mid f)$ are assumed as follows

$$
\begin{aligned}
& S(f)=\sum_{i} \alpha_{i} H_{1 / 3_{i}}^{2} T_{p i}\left(f / f_{p i}\right)^{-m_{i}} \\
& \exp \left\{-\left(m_{i} / n_{i}\right)\left(f / f_{p i}\right)^{-n_{i}}\right\} \gamma_{i}^{\exp \left\{-\left(f / f_{p i}-1\right)^{2} / 2 \sigma^{2}\right\}}
\end{aligned}
$$

where, $\quad f_{p}=\left[1-0.132(\gamma+0.2)^{-0.559}\right] / T_{1 / 3}$,

$$
\begin{gathered}
\sigma=\left\{\begin{array}{cc}
0.07 & \left(0.7 f_{p}<f<f_{p}\right) \\
0.09 & \left(f_{p} \leq f<f_{p}\right)
\end{array}\right. \\
G(\theta \mid f)=\sum_{i} \beta_{i} \cos ^{2 S_{i}}\left\{\left(\theta-\bar{\theta}_{i}\right) / 2\right\}
\end{gathered}
$$

where, $S=\left\{\begin{array}{cc}S_{\max }\left(f / f_{p}\right)^{5} & \left(f<f_{p}\right) \\ S_{\max }\left(f / f_{p}\right)^{-2.5} & \left(f \geq f_{p}\right)\end{array}\right.$

For the simulations of unimodal directional spectrum, the suffix $i$ is with $i=1$ only, while two different wave groups with different sets of parameters, $H_{1 / 3}, T_{1 / 3}, \gamma_{i}, S_{i}, \theta_{i}:(i=2)$, are superposed for bimodal directional spectrum. $\alpha_{i}$ and $\beta_{i}$ are propotionality coefficients. 


\section{(2) Directional energy distributions with various energy concentration parameters $S_{\max }$ and $\gamma$}

In this section, directional energy distribution, $E(\theta)$, is defined as an integrated value of directional spectrum, $S(f)$, with respect to the frequency $f$. Figure 1 shows examples of $E(\theta)$ computed with various energy concentration parameters $S_{\max }$ and $\gamma$ listed in Table 1. The dash lines show the initial value of $E(\theta)$, while the soid lines show the ones after 12 hour simulations. As seen in Fig. 1, after 12 hours, the directional peak energy decreases and the directional energy distribution spreads in each case. Although the changes of $E(\theta)$ are similar in three methods, DIA is most diffusive than the others. SRIAM is a little diffusive than RIAM.

\section{(3) Directional distribution of nonlinear energy transfer with various energy concentration parameters $S_{\max }$ and $\gamma$}

Figure 2 shows the directional energy distributions of the nonlinear energy transfer computed for various energy concentration parameters. As seen in Fig. 2, the directional distributions of the nonlinear energy transfer always show 2 positive lobes and 1 negative lobe pattern.
Journal of Japan Society of Civil Engineers, Ser. B3 (Ocean Engineering), Vol. 71, No. 2, I_347-I_352, 2015.

The magnitude of the peak absolute value of directional distribution increases as the energy concentration $S_{\max }$ increases in all the cases. Comparing differences among three methods, the magnitude of nonlinear transfer is in the order of RIAM > SRIAM > DIA when the energy concentration parameters $S_{\max }$ are large. While, the magnitude of nonlinear transfer of DIA is larger than the others when the energy concentration parameter is low. These characteristics of DIA, RIAM, and SRIAM seem to lead a little difference as seen in Fig.1.

Table 1. Combinations of energy concentration parameters $\gamma$ and $S_{\max }$ used in numerical simulation

\begin{tabular}{ccc}
\hline$j$ & Smax & $y$ \\
\hline 1 & 10 & 1.0 \\
2 & 10 & 3.3 \\
3 & 10 & 8.0 \\
4 & 25 & 1.0 \\
5 & 25 & 3.3 \\
6 & 25 & 8.0 \\
7 & 75 & 1.0 \\
8 & 75 & 3.3 \\
9 & 75 & 8.0 \\
\hline
\end{tabular}

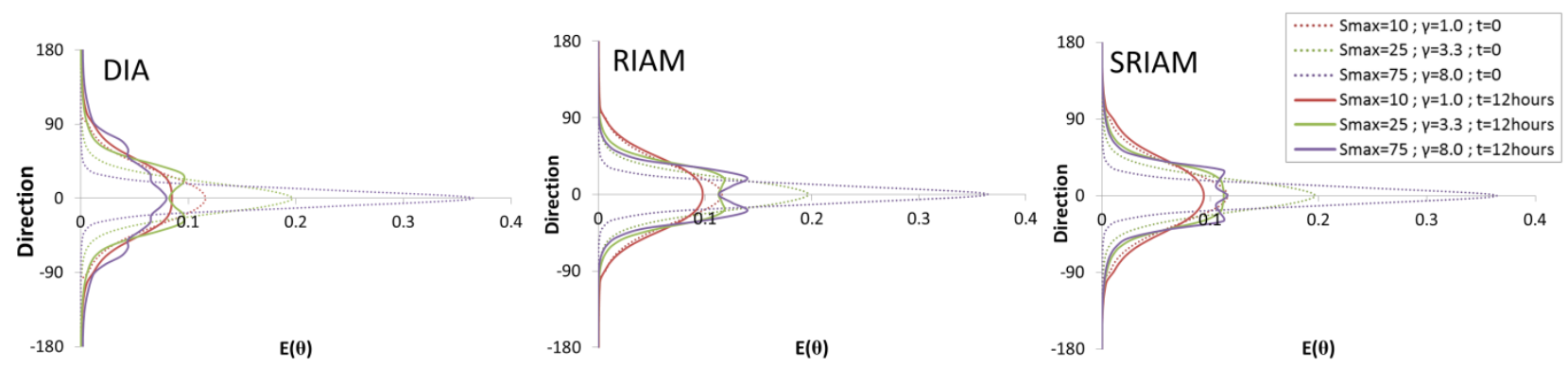

Fig. 1 Directional energy distribution with various energy concentration parameters, $\gamma$ and $S_{\max }$, computed by WAM implemented with DIA, RIAM, and SRIAM
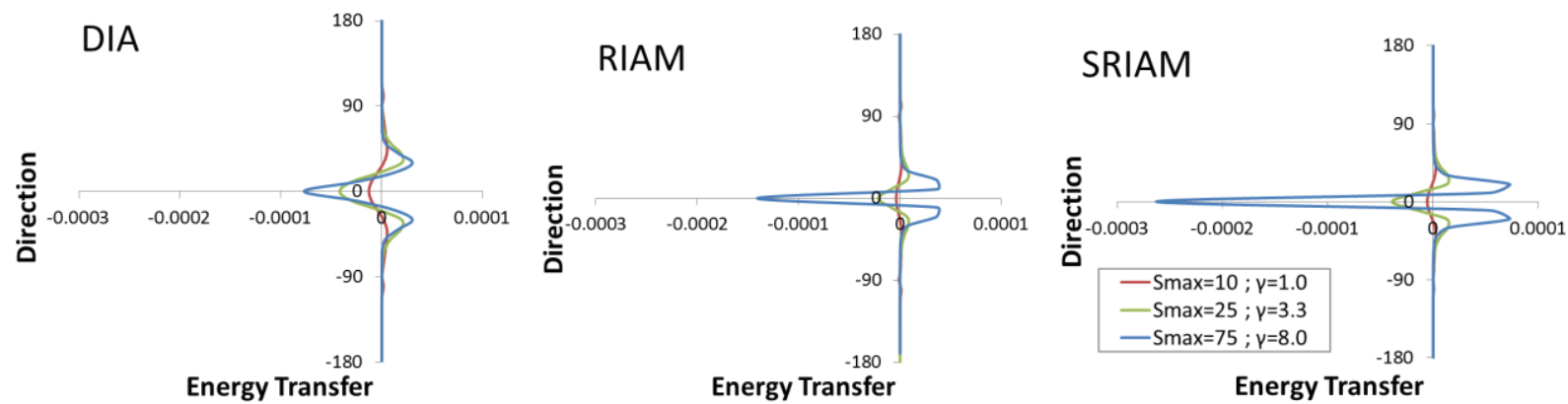

Fig. 2 Directional distribution of nonlinear energy transfer with various energy concentration parameters, $\gamma$ and $S_{\max }$, computed by WAM implemented with DIA, RIAM, and SRIAM 


\section{(4) Bimodal directional spectra with various crossing angle $\Delta \theta$.}

Characteristics of the nonlinear energy transfer are investigated for bimodal directional spectra based on numerical results computed by SRIAM since SRIAM seems to be superior in efficiency and accuracy.

Figure 3 (a-1) shows a bimodal directional spectrum, where their peak frequencies are at $f_{p}=0.13 \mathrm{~Hz}$ and $f_{p}=0.1 \mathrm{~Hz}$, respectively, with the crossing angle between their propagation directions is $0^{\circ}$. This bimodal spectrum is used as the initial condition for duration-limited simulation. While, (a-2) shows the directional spectrum after 12 hours. As seen in these figures, the energy distribution gradually changes into a unimodal wave spectrum. The higher frequency peak disappears and the directional spectrum becomes broader as seen in (a2). Figure 3 (b) and (c) show the frequency spectrum and the integrated directional function with respect to frequency $f$, respectively. The blue line shows the initial bimodal spectrum, where the energy concentration parameters are assumed as $\gamma=3.3$ and $S_{\max }=15$ in higher peak frequency spectrum, and $\gamma=7.0$ and $S_{\max }=75$ in the lower frequency one. The red line shows the results after 12 hours of simulation. The shape of the spectrum changes into unimodal one with frequency downshift. While, the shape of the directional function changes into wider distribution.
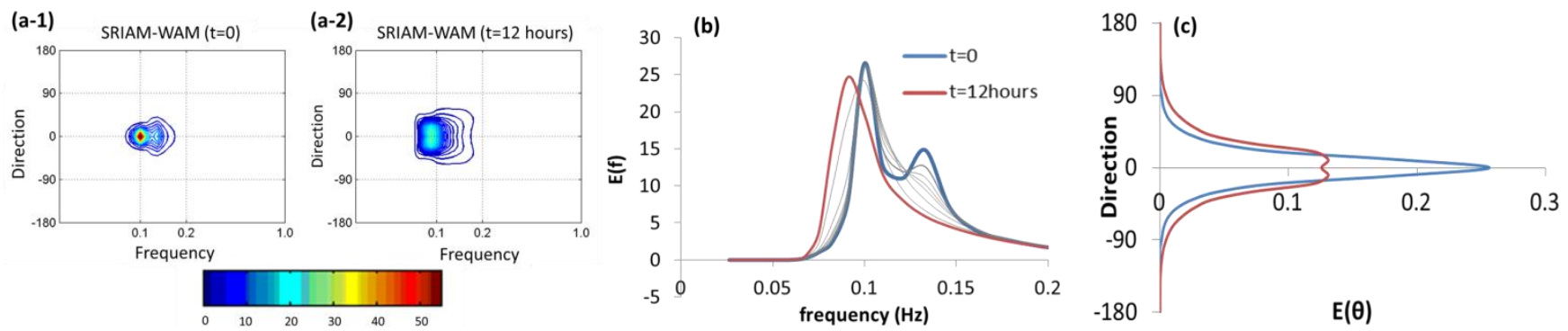

Fig. 3 The directional spectra of bimodal directional spectrum with crossing angle $\Delta \theta=0^{\circ}$ at $t=0$ (a-1), $t=12$ hours (a-2), the temporal change of frequency spectra (b) and directional functions (c)
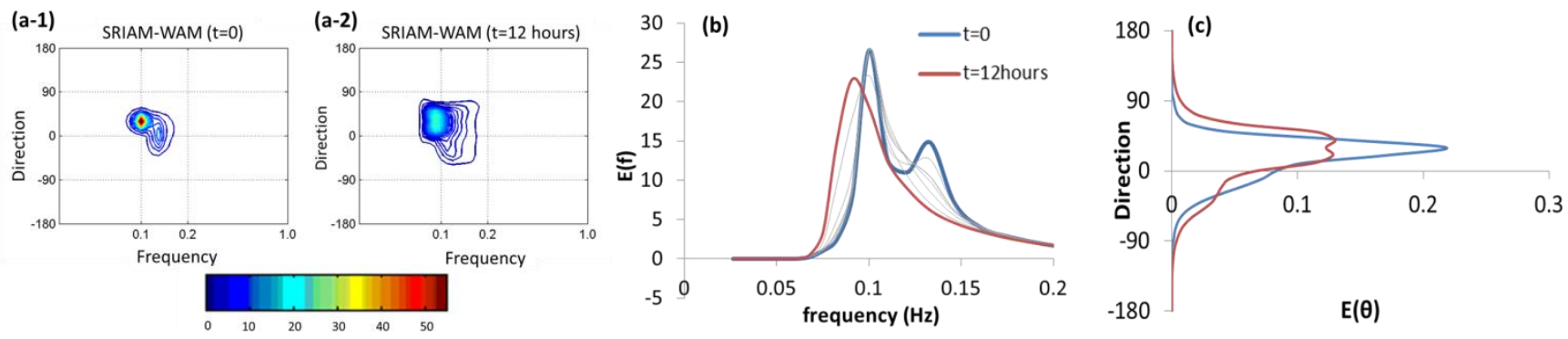

Fig. 4 The directional spectra of bimodal directional spectrum with crossing angle $\Delta \theta=30^{\circ}$ at $t=0$ (a-1), $t=12 \mathrm{hours}$ (a-2), the temporal change of frequency spectra (b) and directional functions (c)
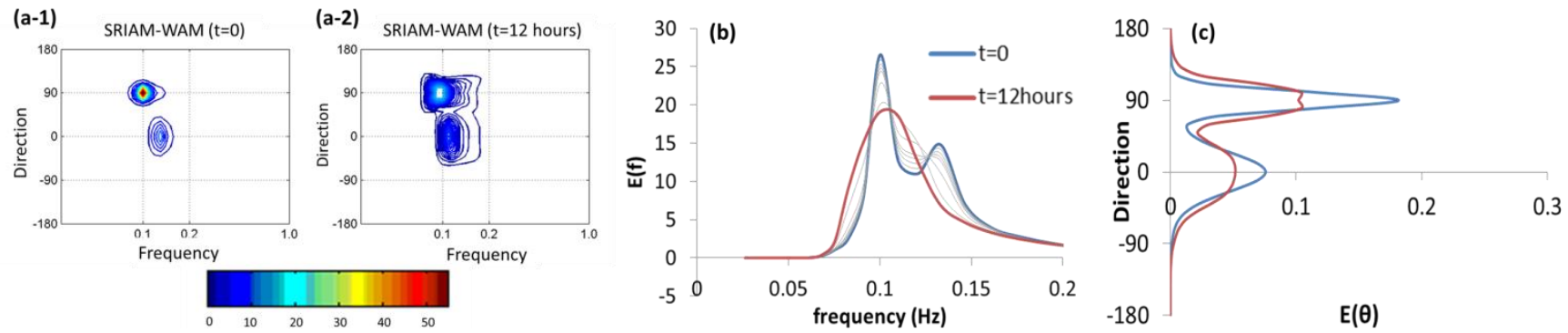

Fig. 5 The directional spectra of bimodal directional spectrum with crossing angle $\Delta \theta=90^{\circ}$ at $t=0$ (a-1), $t=12$ hours (a-2), the temporal change of frequency spectra (b) and directional functions (c) 


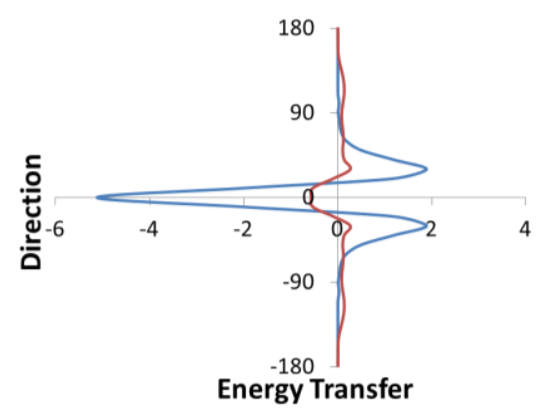

(a)

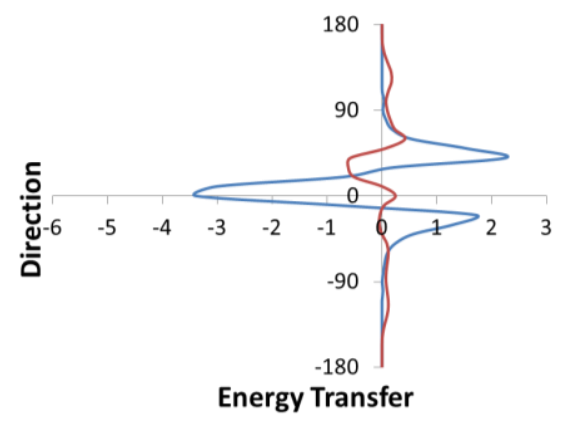

(b)

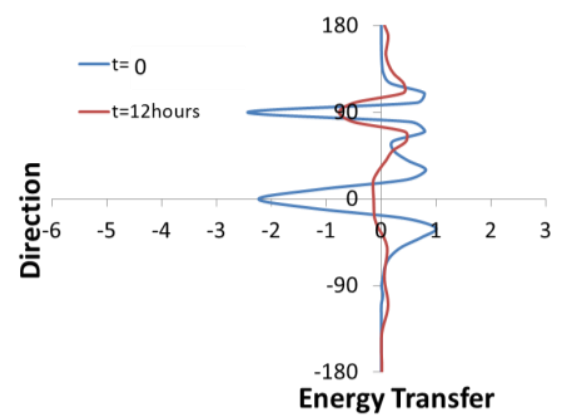

(c)

Fig. 6 Directional disributions of the nonlinear energy transfer with crossing angle (a) $0^{\circ}$, (b) $30^{\circ}$, and (c) $90^{\circ}$

Figure 4 (a-1), (a-2), (b) and (c) show similar results shown in Fig. 3, where a difference is the crossing angle of $30^{\circ}$ between two wave groups. While, Fig. 5 (a-1), (a-2), (b) and (c) also show the similar results with the crossing angle of $90^{\circ}$ between two wave groups. As seen in (b) in Fig. 4 and $\mathbf{5}$, after 12 hours simulations, both frequency spectra change into unimodal ones. However, although the frequency spectrum (b) in Fig. 4 show comparably large frequency downshift as seen in Fig.3 (b), Fig. 5 (b) does not show clear frequency downshift. While, the directional function (c) in Fig. 5 still shows clear bimodal peaks with directional energy diffusion even after 12 hours. The differences seen in Fig. 3, 4, and 5 seem to arise from the difference in the intensity of the nonlinear energy transfer depending on the crossing angle between two wave groups.

Figure 6 (a), (b), and (c) show the directional distribution of the integrated nonlinear energy transfer with respect to frequency $f$, where (a), (b), and (c) correspond to Fig. 3, 4, and 5, respectively. The blue lines show the results at $t=0 \mathrm{~s}$, while the red lines show the ones at $t=12$ hours, respectively. Figure 6 (a) shows the symmetry distribution having two positive lobes and one negative lobe, with gradually reducing intensity of the nonlinear energy transfer over the time. Figure 6 (b) and (c) show asymmetry distribution. However, comparing (c) with (a) and (b), seems to have different characteristics, i.e., the directional distribution of the negative and positive lobes around the two peak frequencies in (c) seems to be rather independent (weak interaction) each other. This may lead to the temporal change of frequency spectra in Fig. 5.

\section{CONCLUSIONS}

This study evaluated the characteristics of the directional characteristics of the nonlinear energy transfer by a third generation wave model, WAM, implemented with DIA, RIAM, and SRIAM. As a result, an interesting characteristic influenced by the crossing angle $\Delta \theta$ between two wave groups in bimodal spectrum is observed. That is, large coupling between them can be seen when the two peak frequencies in bimodal spectrum is relatively narrow in direction.

ACKNOWLEDGMENT: This work was supported by JSPS KAKENHI Grant Number 26289166.

\section{REFERENCES}

1) Hasselmann, K., 1962, On the non-linear energy transfer in a gravity-wave spectrum, Part 1, General theory, Journal of Fluid Mechanics, 12, 481-500.

2) Hasselmann, K. et al., 1973, Measurements of wind waves growth and swell decay during the Joint North Sea Wave Project (JONSWAP), Dt. Hydrogr. Z., A8(12), 95p.

3) Hasselmann, S. and K. Hasselmann, 1985, Computations and Parameterizations of the Nonlinear Energy Transfer in a Gravity-Wave Spectrum. Part I: A New Method for Efficient Computations of the Exact Nonlinear Transfer Integral, Journal of Physical Oceanography, 15, 1378-1391.

4) Komatsu, K., T. Kusaba and A. Masuda, 1993, An efficient method for computing nonlinear energy transfer among wind waves, Bull. Res. Inst. Appl. Mech. Kyushu Univ., 75, 121-146 (in Japanese).

5) Komatsu, K. and A. Masuda, 1996, A new scheme of nonlinear energy transfer among wind waves: RIAM method - algorithm and performance -, Journal of Oceanography, 52, 509-537.

6) Komatsu, K. 1996, Development of a new generation wave forecasting model based on a new scheme of nonlinear energy transfer among wind waves, Dr. Thesis, University of Kyushu, 155p. (in Japanese).

7) Masuda, A, 1980, Nonlinear energy transfer between wind waves, Journal of Physical Oceanography, 10, 2082-2092.

8) Resio, D. and W. Perrie, 1991, A numerical study of nonlinear energy fluxes due to wave-wave interactions, Journal of Fluid Mechanics, 223, 603-629. 